\title{
Superresolution STED imaging reveals a periodic punctate pattern of adenylyl cyclase type III on primary cilia
}

\author{
J Liao*, T Yang, P Hampilos \\ From First International Cilia in Development and Disease Scientific Conference (2012) \\ London, UK. 16-18 May 2012
}

\begin{abstract}
Adenylyl cyclases type III (ACIII) is a primary cilia marker involved in cAMP signaling, playing important roles in regulating ciliogenesis and sensory function. Despite its importance, detailed ACIII localization and their interactions with other proteins remain unclear due to the limited resolution of conventional microscopy. To determine the morphological characteristics of ACIII in primary cilia, we conducted superresolution imaging of immunostained ACIII in fibroblasts and neurons using stimulated emission depletion (STED) microscopy, which allows us to resolve the localization of ACIII achieving a resolution of $50 \mathrm{~nm}$. In contrast to the previous understanding that ACIII distributes uniformly along a primary cilium, our STED images revealed that ACIII formed a periodic punctate pattern with a roughly equal spacing between groups of puncta. These puncta occupied less than $50 \%$ of the area, with the size of $137 \pm 20 \mathrm{~nm}$ in the axial direction along the primary cilia. The spacing between puncta was $250 \pm 67 \mathrm{~nm}$. Some primary cilia even showed two rows of periodic puncta along the axial direction, with a tilted angle of about $12^{\circ}$ to $35^{\circ}$ between the two rows. The spacing between the two rows was $195 \pm 19 \mathrm{~nm}$. In some cells, ACIII was only localized in the basal body, where the periodic punctate pattern was absent. In summary, based on our superresolution studies, we found that ACIII can be transported into a primary cilium, but would only occupy regions approximately equally spaced along the cilium.
\end{abstract}

Published: 16 November 2012

* Correspondence: jcliao@columbia.edu Columbia University, NY, USA

( 2012 Liao et al; licensee BioMed Central Ltd. This is an Open Access article distributed under the terms of the Creative Commons Attribution License (http://creativecommons.org/licenses/by/2.0), which permits unrestricted use, distribution, and reproduction in any medium, provided the original work is properly cited.
doi:10.1186/2046-2530-1-S1-P32

Cite this article as: Liao et al: Superresolution STED imaging reveals a periodic punctate pattern of adenylyl cyclase type III on primary cilia. Cilia 2012 1(Suppl 1):P32.

Submit your next manuscript to BioMed Central and take full advantage of:

- Convenient online submission

- Thorough peer review

- No space constraints or color figure charges

- Immediate publication on acceptance

- Inclusion in PubMed, CAS, Scopus and Google Scholar

- Research which is freely available for redistribution 